\title{
原子层沉积氧化铝包覆羰基铁粉的抗腐蚀性及吸波性能
}

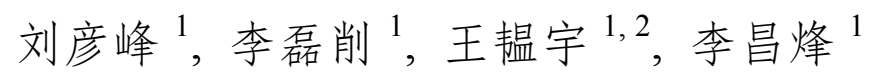

(1. 江苏大学 能源与动力工程学院, 镇江 212013; 2. 镇江智联德科技有限公司, 镇江 212000)

摘 要: 为了提高羰基铁粉的抗腐蚀能力及改善其电磁性能，以 $\mathrm{TMA}$ 和 $\mathrm{H}_{2} \mathrm{O}$ 为前驱体，利用原子层沉积(ALD)方法 对羰基铁粉进行表面包覆改性，在羰基铁粉表面包覆不同厚度的氧化铝。通过扫描电镜(SEM)、透射电镜(TEM)、 综合热分析仪(TGA)、红外光谱(FTIR)和矢量网络分析仪等技术手段系统分析了改性前后羰基铁粉性能指标。结果 表明，通过 ALD 方法可在羰基铁粉表面生长纳米级别具有良好保型的氧化铝薄膜，形成了极佳的羰基铁/氧化铝壳 层结构复合材料。与原样品相比，包覆改性后的羰基铁粉热稳定性与抗腐蚀性有极大的提高，且随着包覆厚度的增 加, 抗氧化能力增强, 最大抗氧化温度可超过 $550^{\circ} \mathrm{C}$ 。同时羰基铁粉包覆氧化铝后, 其介电常数明显减小, 磁导率 变化相对较小, 改善了原羰基铁粉的电磁参数与吸波性能。

关 键 词: 原子层沉积; 羰基铁粉; 氧化铝; 吸波材料; 抗腐蚀性

中图分类号: TB33 文献标识码: A

\section{Corrosion Resistance and Wave Absorbing Property of Carbonyl Iron Powder Coating with Alumina by Atomic Layer Deposition}

\author{
LIU Yan-Feng $^{1}$, LI Lei-Xiao ${ }^{1}$, WANG Yun-Yu ${ }^{1,2}$, LI Chang-Feng ${ }^{1}$
}

(1. School of Energy and Power Engineering, Jiangsu University, Zhenjiang 212013, China; 2. ZLD technology Co. Ltd, Zhenjiang, Zhenjiang 212000, China)

\begin{abstract}
To improve the corrosion resistant capability and electromagnetic performance of carbonyl iron powders (CIPs), alumina thin films were coated on carbonyl iron powder by atomic layer deposition (ALD) using trimethylaluminum and water as precursors. Original and modified carbonyl iron powder was characterized comprehensively via scanning electron microscope (SEM), transmission electron microscope (TEM), thermogravimetric analyzer (TGA), Fourier Transform infrared spectroscope (FTIR), and vector network analyzer. The results indicated that nano-alumina films were conformably grown on the surface of carbonyl iron powders via ALD, forming an excellent core-shell CIP/alumina structure. Compared to the original sample, thermal stability and corrosion resistance of the modified carbonyl iron powders were greatly improved. Antioxidation capability of CIPs was found enhanced with the increase of alumina coating thickness, and the maximum antioxidation temperature can exceed $550^{\circ} \mathrm{C}$. It was also found that the complex permittivity of core-shell CIP/alumina decreased greatly while the complex permeability was almost the same. Therefore, the electromagnetic parameters and microwave absorption properties of the modified CIPs was also improved.
\end{abstract}

Key words: atomic layer deposition; carbonyl iron powder; alumina; wave absorbing materials; corrosion resistant

收稿日期: 2016-10-08; 收到修改稿日期：2016-11-21

基金项目：国家自然科学基金(11072091)；江苏省“青蓝工程”项目；江苏省镇江市“331 工程”项目 National Natural Science Foundation of China (11072091); 'Qing Lan' Project Foundation of Jiangsu Province; Zhenjiang '331 Project', Jiangsu Province

作者简介: 刘彦峰(1990-), 男, 硕士研究生. E-mail: yanfengf@163.com

通讯作者: 李昌烽, 教授.E-mail: cfli@ujs.edu.cn 
近年来, 吸波材料在军事和民用领域的用途越 来越广泛, 这引起了广泛关注。吸波材料按吸波原 理不同可分为磁损耗型与电损耗型, 常见的吸波剂 有羰基铁粉(carbonyl iron particles, CIP)、铁氧体、 钛酸钡等, 其中羰基铁粉具有磁导率高, 吸波频带 宽, 吸波效果好等诸多优点 ${ }^{[1-2]}$, 成为国内外应用最 为广泛的吸波材料之一, 并成功应用于先进军事装 备中 ${ }^{[3-4]}$ 。然而, 由于铁是一种比较活泼的金属, 容 易与空气和水反应，使其在使用过程中出现腐蚀、 氧化等诸多问题, 并对它的吸波性能产生较大影响, 尤其在高温潮湿环境下上述问题尤为突出, 这也限 制了它在这些特殊环境下的应用。

为了解决上述问题, 很多学者做了大量的研究, 利用不同的薄膜生长方法对颗粒表面包覆, 制备核 壳结构复合材料, 提高羰基铁粉的抗氧化性、抗腐蚀 性并改善电磁参数。例如 $\mathrm{Zhu}$ 等 $^{[5]}$ 通过溶胶-凝胶法 在羰基铁粉表面包覆 $\mathrm{SiO}_{2}$ 纳米薄膜, 抗氧化性和抗 腐蚀性都得到明显的提高, 同时电阻率和电磁参数 也有所改善。周乾等 ${ }^{[6]}$ 用相同的方法也得到类似结 果。Zhou 等 ${ }^{[7]}$ 用电镀方法制备了钴包覆的羰基铁颗 粒, 将氧化起始温度升高到 $300^{\circ} \mathrm{C}$, 显示出良好的 热稳定性。 $\mathrm{Li}$ 等 $^{[8]}$ 用电镀法制备了镍一嗍/羰基铁复 合材料，抗氧化性和吸波能力都有所提高。 Abshinova 等 ${ }^{[9]}$ 在羰基铁表面包覆聚苯胺形成核壳结 构, 抗氧化性和吸波性能都有所改善。郭飞等 ${ }^{[10]}$ 利用 水热法制备海胆状氧化锌羰基铁粉复合材料, 着重 研究了氧化锌壳对吸波性能及抗氧化性能的影响。

原子层沉积(Atomic layer deposition, ALD)是一 种纳米薄膜生长技术 ${ }^{[11]}$, 具有其他薄膜制备方法难 以媲美的优点, 如包覆均匀、薄膜厚度精确可控、 高保型性、可生长超薄薄膜等, 在粉体材料包覆领 域具有广阔的应用前景。目前，原子层沉积(ALD) 技术在铜、镁、钙等金属防腐领域的研究已取得一 些成果 ${ }^{[12-14]}$, 但是对包覆超细金属粉末的报道尚不 多见。本工作采用原子层沉积(ALD)法, 以 TMA 和 水为前驱体, 在羰基铁粉表面包覆纳米氧化铝薄膜, 制备具有优良热稳定性、抗腐蚀性以及电磁性能的 羰基铁粉/氧化铝 $\left(\mathrm{CIP} / \mathrm{Al}_{2} \mathrm{O}_{3}\right)$ 核壳结构复合材料。

\section{1 实验方法}

\section{1 实验材料}

微米级羰基铁粉由江苏天一超细金属粉末有限公 司提供, 粒径 2 5 $\mu \mathrm{m}$, 实验室自制去离子水 $(5 \mathrm{M} \Omega \cdot \mathrm{cm})$, 异丙醇(国药集团), 浓盐酸(国药集团), 前驱体为高
纯水和 TMA。

\section{2 羰基铁/氧化铝复合材料制备}

ALD 沉积系统采用江苏镇江智联德科技有限 公司的 Twinstar 型 ALD 颗粒研发系统，具体操作步 骤如下: 取 $30 \mathrm{~g}$ 羰基铁粉放入 $500 \mathrm{~mL}$ 烧杯, 用去 离子水清洗 3 次, 超声 $30 \mathrm{~min}$, 抽滤后将得到的样 品按上述步骤用异丙醇清洗, 然后将羰基铁置于真 空干燥箱, $50^{\circ} \mathrm{C}$ 烘烤 $10 \mathrm{~h}$, 得到干燥洁净的样品。用 异丙醇清洗反应腔室, 然后放入样品, 抽取真空, 最终腔室气压维持在 $6.65 \mathrm{~Pa}$ 。利用 $\mathrm{TMA}$ 与 $\mathrm{H}_{2} \mathrm{O}$ 做 前驱体时, 氧化铝薄膜生长分为两步 ${ }^{[15]}$ :

(1) 通入 TMA，与羰基铁粉表面-OH 反应生成 单层 $\mathrm{Al}\left(\mathrm{CH}_{3}\right)_{x}$ 基团与副产物 $\mathrm{CH}_{4}$, 利用 $\mathrm{N}_{2}$ 吹扫多余 反应气体;

（2）通入 $\mathrm{H}_{2} \mathrm{O}, \mathrm{Al}\left(\mathrm{CH}_{3}\right)_{x}$ 基团与 $\mathrm{H}_{2} \mathrm{O}$ 反应，将表 面的 $-\mathrm{CH}_{3}$ 再次置换成 $-\mathrm{OH}$, 释放出 $\mathrm{CH}_{4}$ 。

总的反应式如下:

$$
2 \mathrm{Al}\left(\mathrm{CH}_{3}\right)_{3}+3 \mathrm{H}_{2} \mathrm{O} \longrightarrow \mathrm{Al}_{2} \mathrm{O}_{3}+3 \mathrm{CH}_{4}
$$

由于羰基铁在高温环境易发生氧化反应, 为了 减少 ALD 反应过程中羰基铁粉自身的氧化作用, 本实验将反应温度控制在 $150^{\circ} \mathrm{C}$ 。将 $\mathrm{H}_{2} \mathrm{O}$ 和 TMA 分 压设置为 $66.5 \mathrm{~Pa}$, 以脉冲形式交替通入腔室, 脉冲 之间用高纯氮( $99.999 \%)$ 吹扫残余气体，分压设为 $2660 \mathrm{~Pa}$ (反应脉冲如图 1 所示)。通过控制循环周期, 可以得到不同厚度的薄膜，实验制取 25、50、75 个 循环厚度的薄膜。

\section{3 样品表征及性能测试}

利用日本电子株式会社生产的 JSM-7001F 扫描 电子显微镜分析包覆前后样品表面形貌，加速电压 $15 \mathrm{kV}$, 美国 FEI 公司透射电镜分析薄膜厚度。使用 德国 NETZSCH 仪器公司STA449C 综合热分析仪进 行热重分析; 配置 $0.2 \mathrm{~mol} / \mathrm{L} \mathrm{HCl}$ 溶液, 做加速腐蚀 实验, 对比包覆后样品耐腐蚀性。采用傅里叶红外 光谱仪(FTIR)分析包覆前后红外光谱吸收特性。通

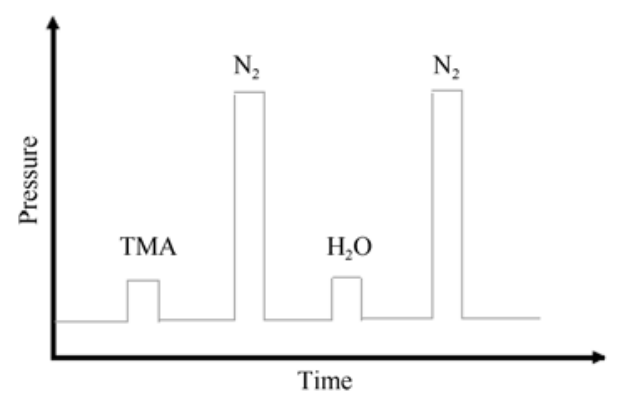

图 1 原子层沉积过程反应脉冲示意图

Fig. 1 Schematic diagram of reaction pulse during ALD 
过等离子体发射光谱仪表征(ICP)样品包覆量。使 用矢量网络分析仪(安捷伦 N5244A)测试样品的电 磁性能, 将样品与石蜡按质量比 4: 1 混合, 制成外 径 $7 \mathrm{~mm}$ 、内径 $3 \mathrm{~mm}$ 、厚度 $2.5 \mathrm{~mm}$ 的同轴环, 测 试频率范围为 $2.0 \sim 18.0 \mathrm{GHz}$ 。

\section{2 结果分析与讨论}

\section{1 表面形貌分析}

图 2 给出了羰基铁粉包覆氧化铝前后的形貌图 及其元素分布特征, 图 2(a)为原羰基铁粉(CIP)的 SEM 形貌，图 2(b) 是经过 75 次 ALD 循环包覆后得 到的氧化铝/羊炭基铁粉 $\left(\mathrm{CIP} / \mathrm{Al}_{2} \mathrm{O}_{3}\right)$ 复合材料的 SEM 形貌, 羰基铁粉具有良好的球形度, 粒径为 $3 \mu \mathrm{m}$ 左 右, 这与厂家提供的数据基本一致。对比包覆前后 形貌可以发现包覆氧化铝后的羰基铁粉表面仍然可 以看到原始轮廓, 这说明 ALD 技术包覆不改变羰 基铁颗粒形貌及团簇。图 2(c)为 $\mathrm{CIP} / \mathrm{Al}_{2} \mathrm{O}_{3}$ 的 TEM 照片, 对区域 1 进行元素分析, 显示出较强的 $\mathrm{O}$ 元 素和 $\mathrm{Al}$ 元素峰(图 2(d)), 可以确认该区域为 $\mathrm{Al}_{2} \mathrm{O}_{3}$ 包覆层。由图 2(c)可以看出, 该包覆层是一种致密 的无定型结构 ${ }^{[18]}$, 厚度大约为 $20 \mathrm{~nm}$, 计算得到其 生长速率为 $0.27 \mathrm{~nm} /$ 次左右, 这说明在低温条件下, ALD 沉积速率较高温时快 ${ }^{[16]}$ 。由图(e) (h) 可知, 对 区域 2 进行 eds-maping 分析, $\mathrm{Al}$ 和 $\mathrm{O}$ 元素信号均匀 分布在所示区域。综上, 羰基铁粉经过 ALD 循环后, 可以形成具有致密、均匀、完全包覆的高质量核壳 结构复合材料。

\subsection{FTIR 及铝含量分析}

图 3 给出了羰基铁粉经过 75 个 ALD 循环包覆 氧化铝前后的红外吸收光谱图, 从图中可以看出, a、 $\mathrm{b}$ 两条曲线在 $650 \mathrm{~cm}^{-1}$ 附近有许多密集的小吸收峰, 可以推测为羰基铁粉特征吸收峰 ${ }^{[17]}$ 。在 $3400 \mathrm{~cm}^{-1}$ 附 近的吸收峰比较宽, 这可能是羟基伸缩振动引起的 吸收峰, 对比包覆前后, 可以发现包覆后羟基吸收 峰减弱, 由 ALD 生长氧化铝原理可知, 反应过程中 必定会消耗掉一部分羰基铁粉表面吸附的羟基。 $2400 \mathrm{~cm}^{-1}$ 附近出现的吸收峰应该是由测试环境中二 氧化碳引起的, 从整个吸收波数范围来看, 包覆前 后吸收峰位置基本一致, 未出现新的吸收峰, 说明 制备的羰基铁粉复合材料 $\left(\mathrm{CIP} / \mathrm{Al}_{2} \mathrm{O}_{3}\right)$ 没有引入新的 有机杂质, 材料纯净度较高。

为了定量分析该复合材料中氧化铝的含量, 用 ICP 测试了不同样品铝元素的含量, 发现经过 ALD 循环包覆的样品铝元素含量急剧增加, 并根据结果 计算出不同 ALD 循环包覆的 CIP $/ \mathrm{Al}_{2} \mathrm{O}_{3}$ 复合材料中 氧化铝的质量分数如图 4 所示, 经过 25、50、75 次 ALD 循环包覆, $\mathrm{Al}_{2} \mathrm{O}_{3}$ 质量分数分别为 $0.37 \mathrm{wt} \%$ 、 $0.81 \mathrm{wt} \% 、 1.24 \mathrm{wt} \%$, 这进一步说明成功制备了氧化 铝包覆型的羰基铁粉复合材料。

\section{3 热稳定性分析}

图 5 给出了原始样品以及包覆不同厚度氧化铝后 的羰基铁粉热重分析曲线, 测试条件为: $50 \mathrm{~mL} / \mathrm{min}$ 的 空气气氛下, 以 $10^{\circ} \mathrm{C} / \mathrm{min}$ 的升温速率将样品从室温 $25^{\circ} \mathrm{C}$ 加热至 $800^{\circ} \mathrm{C}$ 。如图 5 所示, 由于高温氧化作用, 四种样品在热分析最后阶段重量都有显著增加,
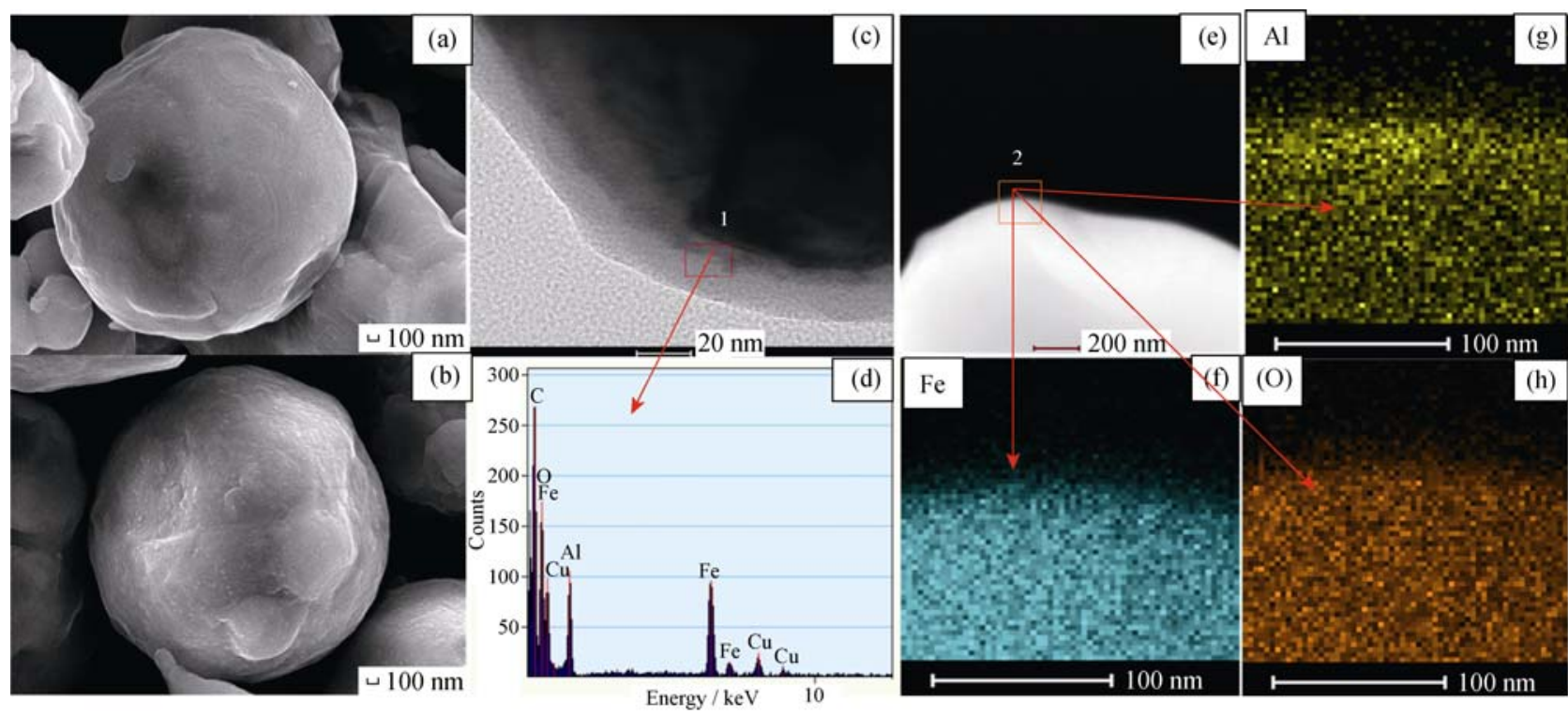

图 $2 \mathrm{CIP}(\mathrm{a})$ 和 $\mathrm{CIP} / \mathrm{Al}_{2} \mathrm{O}_{3}$ (b)的扫描电镜照片; (c $\left.\sim \mathrm{h}\right) \mathrm{CIP} / \mathrm{Al}_{2} \mathrm{O}_{3}$ 的透射电镜照片及能谱图

Fig. 2 SEM images of CIP(a) and CIP/ $/ \mathrm{Al}_{2} \mathrm{O}_{3}(\mathrm{~b})$; (c-h) TEM and EDS of CIP/ $/ \mathrm{Al}_{2} \mathrm{O}_{3}$ 


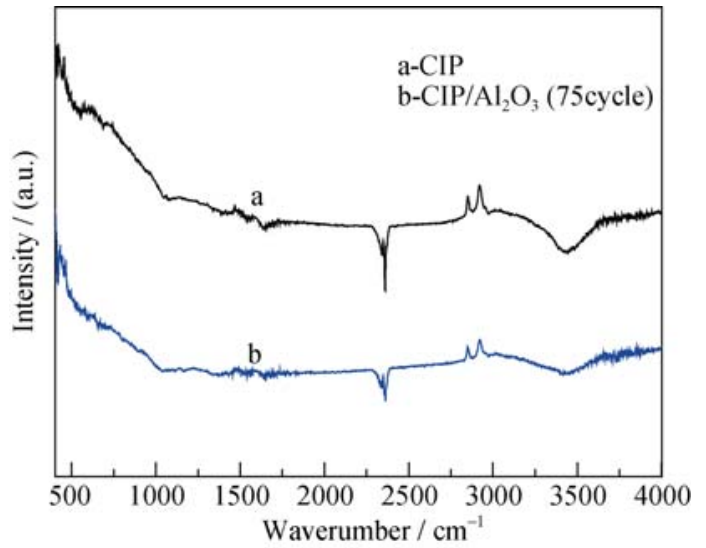

图 $3 \mathrm{CIP}$ 和 $\mathrm{CIP} / \mathrm{Al}_{2} \mathrm{O}_{3}$ 复合材料红外光谱图

Fig. 3 FTIR spectra of (a) CIP and (b) $\mathrm{CIP} / \mathrm{Al}_{2} \mathrm{O}_{3}$

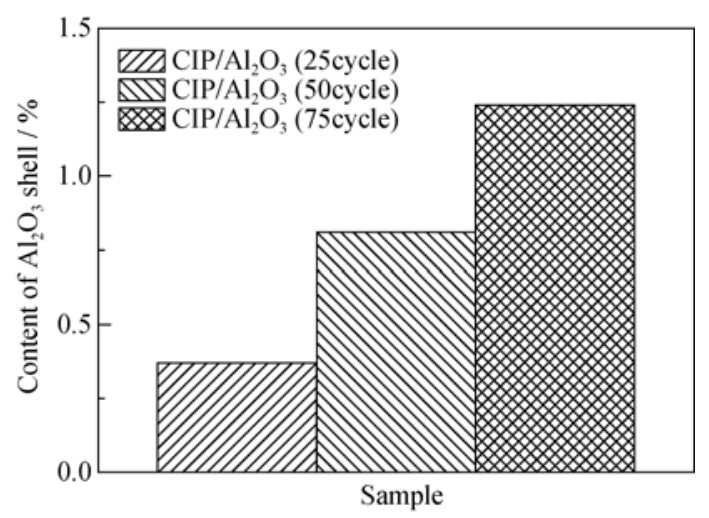

图 4 不同 $\mathrm{ALD}$ 循环包覆的 $\mathrm{CIP} / \mathrm{Al}_{2} \mathrm{O}_{3}$ 复合材料中氧化铝的 质量分数

Fig. 4 Mass fraction of alumina in $\mathrm{CIP} / \mathrm{Al}_{2} \mathrm{O}_{3}$ composites after different cycles of ALD deposition

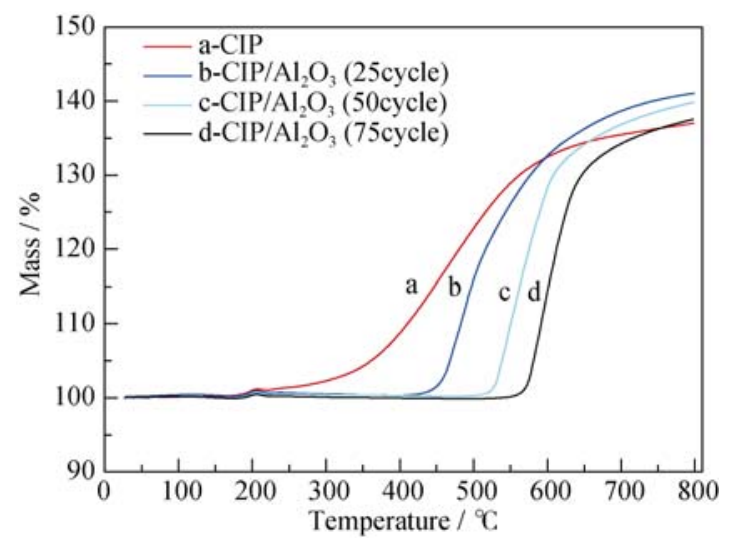

图 $5 \mathrm{CIP}$ 和 $\mathrm{CIP} / \mathrm{Al}_{2} \mathrm{O}_{3}$ 复合材料的热重曲线

Fig. 5 TG curves of CIP and $\mathrm{CIP} / \mathrm{Al}_{2} \mathrm{O} 3$

增加量达到 $40 \%$ 左右, 此时羰基铁粉被氧化成 $\mathrm{Fe}_{2} \mathrm{O}_{3}$ 或 $\mathrm{Fe}_{3} \mathrm{O}_{4}{ }^{[5]}$ 。从氧化过程来看, 四种样品最初 都保持一个较为稳定的平直曲线, 在 $200^{\circ} \mathrm{C}$ 时原羰 基铁粉重量开始增加, 发生氧化反应，而经过 25 、 50、75 次 ALD 循环包覆的复合羰基铁粉样品
$\left(\mathrm{CIP} / \mathrm{Al}_{2} \mathrm{O}_{3}\right)$, 其氧化起始温度分别在 $430^{\circ} \mathrm{C} 、 505^{\circ} \mathrm{C}$ 、 $560^{\circ} \mathrm{C}$ 。相对于原样品氧化起始温度分别升高了 $220^{\circ} \mathrm{C} 、 205^{\circ} \mathrm{C} 、 360^{\circ} \mathrm{C}$, 也就是说羰基铁粉经过氧化 铝包覆后，抗氧化性得到提高，表现出优异热稳定 性, 且随着循环包覆次数的增加, 其热稳定性越好。 这是由于氧化铝是一种比较稳定的物质, 羰基铁粉 经过 ALD 循环包覆后, 在其表面生长一层较为稳 定的致密氧化铝薄层, 在一定程度上能够阻止氧气 与羰基铁发生氧化反应。当升温到一定程度后，保 护层将被破坏, 这时羰基铁粉迅速被氧化, 这就形 成了较为陡峭的变化曲线(曲线 b、c、d)。

\section{4 酸腐蚀测试}

为了测试包覆前后羰基铁粉对酸性环境的耐腐 蚀情况 ${ }^{[5]}$, 室温下分别称取 $0.8 \mathrm{~g}$ 原样品和经过 75 次 ALD 循环包覆后的羰基铁粉样品置于 $0.2 \mathrm{~mol} / \mathrm{L}$ $\mathrm{HCl}$ 的溶液中并用玻璃棒快速摚拌均匀。图 6 显示 了两种样品与盐酸溶液反应情况, 从图 6(a)可以看 出, 在刚刚加入盐酸溶液时, 两种样品溶液分别混 合, 溶液呈浑浊状, 随后发现原样品(CIP)与盐酸溶 液快速反应, 使溶液变得较为浑浊, 并伴随大量气 泡产生，这是由于铁单质与盐酸中的氢离子反应产 生的氢气形成的, 而氧化铝包覆过的样品 $\left(\mathrm{CIP} / \mathrm{Al}_{2} \mathrm{O}_{3}\right)$ 在盐酸溶液中比较稳定, 几乎不与溶液 反应, 未看到明显气泡, 样品随时间延长慢慢地沉 淀至烧杯底部(图 6(b))。这是由于 ALD 生长的氧化 铝薄膜具有比较致密的结构, 可以在一定程度上阻 碍氢离子过薄层, 减弱与羰基铁粉反应。但需要说 明的是氧化铝对氢离子的阻碍并不是持久的, 随着 时间的推移, 包覆样品也逐渐溶解并产生极少量气 泡。经过 $15 \mathrm{~h}$ 腐蚀实验后, 两种溶液都变成黄褐色, 原样品已经被酸腐蚀殆尽，而包覆的羰基铁 $\left(\mathrm{CIP} / \mathrm{Al}_{2} \mathrm{O}_{3}\right)$ 仍然有微量的气泡产生, 还有少量剩余 (图 6(c))。这可能是由于在低于 $400^{\circ} \mathrm{C}$ 的条件下, ALD 制备的氧化铝属于无定型态 ${ }^{[18]}$, 而无定型的氧化铝 可以与强酸发生化学反应。当包覆层被破坏后, 盐 酸溶液同样会与羰基铁粉反应。以上分析可知，包 覆氧化铝的羰基铁粉相对原铁粉，抗腐蚀时间延长 了, 抗腐蚀性得到了提高。

\section{5 复合介电常数与磁导率}

图 7 给出了 75 次 ALD 循环包覆前后羰基铁粉 复介电常数与磁导率随频率的变化关系。从图 7(a) 可以看出, 包覆氧化铝后的羰基铁粉 $\left(\mathrm{CIP} / \mathrm{Al}_{2} \mathrm{O}_{3}\right)$ 介 电常数的实部和虚部都有比较大的下降, 这是因为 氧化铝具有优良的电绝缘性, 包覆的氧化铝层可以 阻碍羰基铁粉电子位移极化, 使得介电常数实部 
(a)

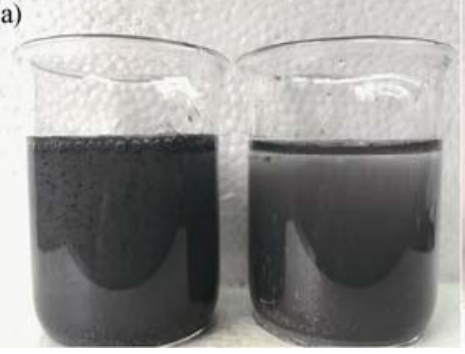

(b)

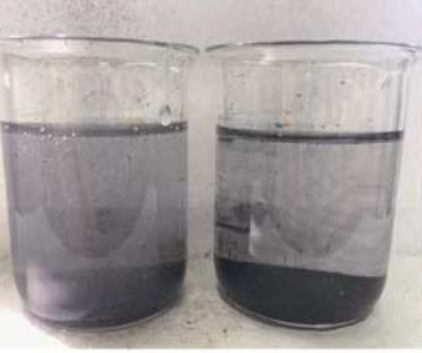

(c)

图 $6 \mathrm{CIP}$ 和 $\mathrm{CIP} / \mathrm{Al}_{2} \mathrm{O}_{3}$ 在 $0.2 \mathrm{~mol} / \mathrm{L} \mathrm{HCl}$ 腐蚀试验

Fig. 6 The corrosion test of CIP and CIP $/ \mathrm{Al}_{2} \mathrm{O}_{3}$ in $0.2 \mathrm{~mol} / \mathrm{L} \mathrm{HCl}$ (a) $2 \mathrm{~min}$; (b) $20 \mathrm{~min}$; (c) $15 \mathrm{~h}$
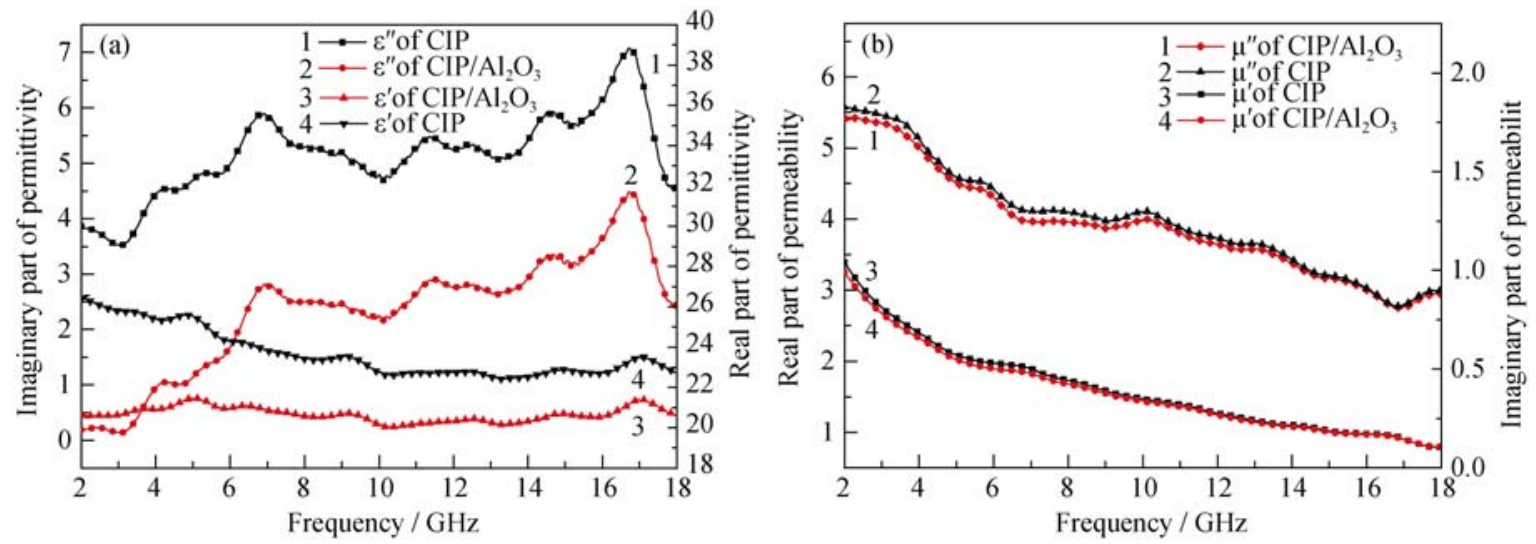

图 $7 \mathrm{CIP}$ 和 $\mathrm{CIP} / \mathrm{Al}_{2} \mathrm{O}_{3}$ 与石蜡混合物的复介电常数与磁导率随频率的变化

Fig. 7 Complex permittivity and permeability relative to the frequency for paraffin mixture of CIP and $\mathrm{CIP} / \mathrm{Al}_{2} \mathrm{O}_{3}$

值 $\varepsilon^{\prime}$ 降低较大。另外包覆不导电氧化铝后也会增大 羰基铁粉的电阻率, 根据自由电子理论介电常数虚 部与电阻率的反比关系: $\varepsilon^{\prime \prime} \approx 1 / 2 \pi \varepsilon_{0} \rho f^{[19]}$, 这会导 致介电常数的虚部下降。而从图 7(b) 可知, $\mathrm{CIP} / \mathrm{Al}_{2} \mathrm{O}_{3}$ 复合磁导率的减小幅度相对较小, 随着频率的增加 呈下降趋势。这是因为羰基铁的磁损耗性和磁导率 主要由材料颗粒大小与内部结构决定 ${ }^{[20]}$ 。从前面 TEM 照片及 FTIR 分析可知, 包覆的氧化铝是一种 纳米级别的薄层, 这几乎不会改变羰基铁的粒径, 也不会影响其内部结构, 但是非磁性的氧化铝引入 会降低羰基铁的质量分数, 由 ICP 分析计算可知, 在 75 次 $\mathrm{ALD}$ 循环包覆后, $\mathrm{Al}_{2} \mathrm{O}_{3}$ 的质量分数达到 $1.24 \mathrm{wt} \%$, 同时考虑到高温下制备样品过程中的氧 化作用, 对样品的磁性会有一些影响, 导致其磁导 率会有一定的下降，降低幅度应为 $>1.24 \%$ 。总之, 羰基铁粉经过 ALD 包覆氧化铝后, 可以调节其电 磁参数, 能够帮助设计吸波屏蔽材料。

\section{6 吸波性能}

根据传输线理论, 利用测量样品的电磁参数可 计算出吸波涂层的反射损耗 ${ }^{[21]}$ :

$$
\begin{gathered}
Z_{\text {in }}=\left(\frac{\mu_{\mathrm{r}}}{\varepsilon_{\mathrm{r}}}\right)^{1 / 2} \tan h\left[j \frac{2 \prod}{c}\left(\mu_{\mathrm{r}} \varepsilon_{\mathrm{r}}\right)^{1 / 2} f d\right] \\
R L(\mathrm{~dB})=20 \lg \left|\frac{Z_{\text {in }}-1}{Z_{\text {in }}+1}\right|
\end{gathered}
$$

式中 $Z_{\text {in }}$ 为归一化的输入阻抗, $\varepsilon_{\mathrm{r}}$ 和 $\mu_{\mathrm{r}}$ 分别是吸波材 料相对复磁导率和介电常数, $f$ 为频率, $c$ 为光速, $d$ 为材料厚度, $R L(\mathrm{~dB})$ 为吸波材料反射损耗。

由公式(2)、(3)模拟计算出了 75 次 ALD 循环 包覆氧化铝前后羰基铁粉反射损耗随频率的变化 关系如图 8 所示，可以看出此款羰基铁粉的吸波范 围在 2 5 GHz，与厂家提供的资料较为符合。原羰 基铁粉(CIP)反射损耗小于 $10 \mathrm{~dB}$ 的频率范围是 $2.42 \sim 3.87 \mathrm{GHz}$, 在 $3.04 \mathrm{GHz}$ 处, 反射损耗最小为 -14.50 ; 包覆后的羰基铁粉反射损耗小于 $10 \mathrm{~dB}$ 的 频率范围为 2.76 4.65 GHz, 在 $3.64 \mathrm{GHz}$ 处出现最 小的反射损耗-20.43 dB, 相对于原样品, 包覆后的 羰基铁粉 $\left(\mathrm{CIP} / \mathrm{Al}_{2} \mathrm{O}_{3}\right)$ 有效吸收范围变宽，反射损耗 峰值向高频移动，峰值降低率达 40.9\%。这是由于 在一般情况下，羰基铁粉具有较大的介电常数，其 实部数值要比磁导率实部大, 包覆氧化铝降低了介 
电常数与磁导率, 但前者降低幅度比后者大，这使材 料的磁导率和介电常数更加接近, 会使得阻抗匹配性 变得更加优良, 这将导致材料具有更小的反射损耗 ${ }^{[6]}$ 。 另一方面, 羰基铁粉包覆氧化铝后可能会使材料的表 面能降低, 减小团聚现象, 即材料变得更加松散, 增 加了气孔率, 改善了透波性能, 进一步增加电磁波在 材料中的散射，使材料能够吸收更多电磁波。 $\mathrm{CIP} / \mathrm{Al}_{2} \mathrm{O}_{3}$ 核壳结构复合材料对电磁波吸收作用示意 如图 9 所示, 由图可见羰基铁粉经过氧化铝的包覆, 可以改善材料的阻抗匹配性, 显示出良好的吸波性 能。一般来说, $\mathrm{CIP} / \mathrm{Al}_{2} \mathrm{O}_{3}$ 核壳结构材料由于其复合效 应使其与纯 CIP 材料对电磁波的作用机理有较大的区 别, 并且核壳结构也可能影响吸波体的介电损耗与磁 损耗, 但其确切作用机理还需进一步研究。

\section{3 结论}

1) 利用原子层沉积(ALD)技术对羰基铁粉进行包 覆改性研究, 成功制备出羰基铁粉/氧化铝 $\left(\mathrm{CIP} / \mathrm{Al}_{2} \mathrm{O}_{3}\right)$

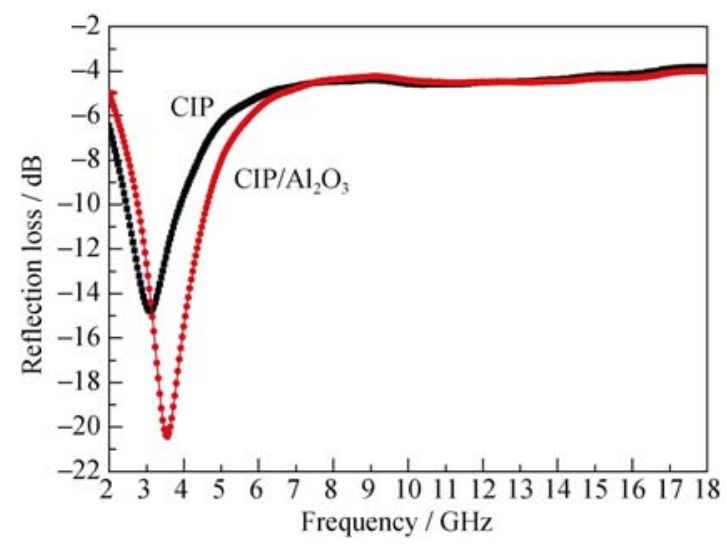

图 $8 \mathrm{CIP}$ 和 $\mathrm{CIP} / \mathrm{Al}_{2} \mathrm{O}_{3}$ 与石蜡混合物的反射损耗频率的变化 $(2.5 \mathrm{~mm})$

Fig. 8 Microwave reflection loss of paraffin mixture for CIP and $\mathrm{CIP} / \mathrm{Al}_{2} \mathrm{O}_{3}(2.5 \mathrm{~mm})$

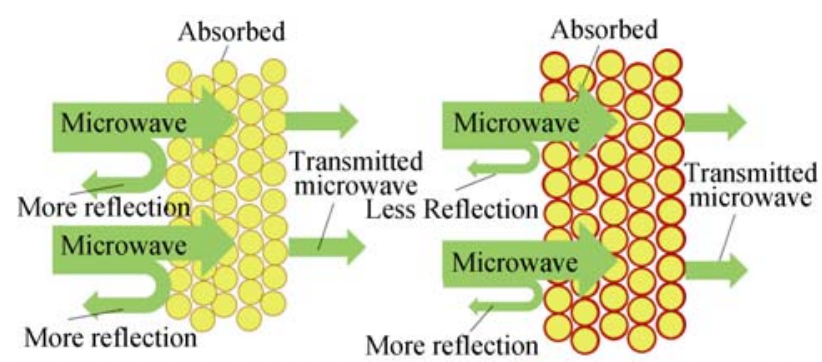

(a) CIP with high permittivity

(b) $\mathrm{CIP} / \mathrm{Al}_{2} \mathrm{O}_{3}$ with low permittivity

图 $9 \mathrm{CIP}(\mathrm{a})$ 和 $\mathrm{CIP} / \mathrm{Al}_{2} \mathrm{O}_{3}(\mathrm{~b})$ 复合材料对电磁波吸收作用示意图 Fig. 9 Schematic diagram of electromagnetic wave absorption of CIP (a) and CIP/ $/ \mathrm{Al}_{2} \mathrm{O}_{3}$ composites (b)
核壳结构复合材料，通过热失重分析，该材料显示 出优良的抗氧化性, 在一定范围内, ALD 循环包覆 次数越多, 其氧化铝包覆层越厚, 抗氧化能力越强; 盐酸测试表明，包覆改性后的羰基铁粉也具有较强 的耐腐蚀性，可保持 $20 \mathrm{~min}$ 以上不发生腐蚀反应。

2) 羰基铁粉经过原子层沉积(ALD)包覆氧化铝 后，电磁参数和吸波性能得到明显的改善，在相同条 件下, 能够得到比原样品更低的反射损耗(-20.43 dB), 降低率达 $40.9 \%$ 。该材料的制备也为 ALD 技术在超 细金属粉末的进一步应用提供了基础。

\section{参考文献:}

[1] LIU JIAN-HUA, ZHOU XIN-MEI, LI SONG-MEI. Electromagnetic properties of carbonyl iron/polyaniline microtubules. Acta Mater. Compos. Sin., 2005, 22(3): 70-74.

[2] MORIHIKO M, MOSHIMORI M. Polymer absorbers contai-ning magnetic particles: effect of polymer permittivityon wave absorption in the quasi microwave band. Journal of Applied Physics, 2002, 91(12): 9635-9637.

[3] LI SHI-TAO, QIAO XUE-LIANG, CHEN JIAN-GUO. Research progress of nano composite wave absorbing materials. Journal of Astronautics. 2006, 2(27): 317-321.

[4] YU M, LI X, GONG R, et al. Magnetic properties of carbonyl iron fibers and their microwave absorbing characterization as the filer in polymer foams. Journal of Alloys and Compounds. 2008, 456(1): 452-455.

[5] ZHU JIAHUA, WEI SUYING, LEE IAN Y, et al. Silica stabilized iron particles toward anticorrosion magnetic polyurethane Nanocomposites. RSC Advances, 2012, 2(3): 1136-1143.

[6] ZHOU QIAN, LU MING. Effect of nano- $\mathrm{SiO}_{2}$ coating on electromagnetic properties of carbonyl iron coating on electromagnetic properties of carbonyl iron Ordnance. Material Science and Engineering, 2013, 36(6): 91-93.

[7] ZHOU YING-YING, ZHOU WAN-CHENG, LI RONG, et al. Enhanced antioxidation and electromagnetic properties of Co-coated flaky carbonyl iron particles prepared by electroless plating. Journal of Alloys and Compounds. 2015, 637: 10-15.

[8] LI RONG, ZHOU WANCHENG, QING YUCHANG. Preparation of Ni-B coating on carbonyl iron and its microwave absorption properties in the X band. Chinese Physics Letters, 2014, 31(9): 097701.

[9] ABSHINOVA MA, KAZANTSEVA NE, SÁHA P, et al. The enhancement of the oxidation resistance of carbonyl iron by polyaniline coating and consequent changes in electromagnetic properties. Polymer Degradation \& Stability, 2008, 93(10): 1826-1831

[10] GUO FEI, DU YUE-LANG, QU SHAO-BO, et al. Oxidation re- 
sistance and microwave absorption property of core shell urchin-like $\mathrm{ZnO} \backslash$ carbonyl iron powder composite particles. Chinese Journal of Inorganic Chemistry, 2015, 31(4): 755-760.

[11] GEORGE. STEVEN M. Atomic layer deposition: an overview. Chemical Reviews. 2009, 110(1): 111-131.

[12] ABDUlagatov A I, YAN Y, COOPER J R, et al. $\mathrm{Al}_{2} \mathrm{O}_{3}$ and $\mathrm{TiO}_{2}$ atomic layer deposition on copper for water corrosion resistance. ACS Applied Materials \& Interfaces, 2011, 3(12): 4593-4601.

[13] WANG P C, SHIH Y T, LIN M C, et al. A study of atomic layer deposited $\mathrm{LiAl}_{x} \mathrm{O}_{y}$ films on $\mathrm{Mg}-\mathrm{Li}$ alloys. Thin Solid Films, 2010, 518(24): 7501-7504.

[14] PARK M, OH S, KIM H, et al. Gas diffusion barrier characteristics of $\mathrm{Al}_{2} \mathrm{O}_{3}$ /alucone films formed using Trimethyl aluminum, water and ethylene glycol for organic light emitting diode encapsulation. Thin Solid Films, 2013, 546: 153-156.

[15] DILlON A C, OTT A W, GEORGE S M, et al. Surface chemistry of $\mathrm{Al}_{2} \mathrm{O}_{3}$ deposition using $\mathrm{Al}\left(\mathrm{CH}_{3}\right)_{3}$ and $\mathrm{H}_{2} \mathrm{O}$ in a binary reaction sequence. Surface Science, 1995, 322(1/2/3): 230-242.

[16] ELAM J W, GRONER M D, GEORGE S M. Viscous flow reactor with quartz crystal microbalance for thin film growth by atomic layer deposition. Review of Scientific Instruments. 2002, 73(8): 2981-2987.

[17] YAO JIN-HUA, YAN HUA, WANG XUE-MEI. Study on surface organic modification of carbonyl iron powder and its effects on properties of magnetorheological fluid. Function Materials, 2010, 10(41): 1684-1690.

[18] MEYER D C, LEVIN A A, PAUFLER P, et al. Crystalline coatings of aluminosilicate $\mathrm{Al}_{2} \mathrm{SiO}_{5}$ polymorphs formed by solid state reaction of alumina layers with silicon/silica substrates. Thin Solid Films, 2005, 489(1): 5-16.

[19] WU HON-JING, WANG LIU-DING, WANG YI-MING, et al. Enhanced microwave performance of highly ordered mesoporous carbon coated by $\mathrm{Ni}_{2} \mathrm{O}_{3}$ nanoparticles. Journal of Alloys \& Compounds, 2012, 525(15): 82-86.

[20] 胡传炘. 隐身涂层技术. 北京: 化学工业出版社, 2004: 8 .

[21] HUANG QI-HUI, ZHANG BAO-SHAN, TANG DONG-MING, et al. Synthesis and characteristics of graphene- $\mathrm{Fe} @ \mathrm{Fe}_{3} \mathrm{O}_{4}$ nano-composites materials. Chinese Journal of Inorganic Chemistry, 2012, 28(10): 2077-2082. 